Menopause26 June 2017

\title{
Effect of programmed exercise on insulin sensitivity in postmenopausal women: a systematic review and meta- analysis of randomized controlled trials
}

\section{( 国 Article in press ? )}

- Bueno-Notivol, J.,

- Calvo-Latorre, J.,

- Alonso-Ventura, V.,

- Pasupuleti, V.,

- Hernandez, A.V.,

- Pérez-López, F.R.,

- the Health Outcomes and Systematic Analyses (HOUSSAY) Project

View Correspondence (jump link)

- 1Facultad de Medicina, Universidad de Zaragoza, Zaragoza, Spain 2ProEd Communications Inc., Cleveland, $\mathrm{OH}$ 3School of Medicine, Universidad Peruana de Ciencias Aplicadas (UPC), Lima, Peru 4University of Connecticut/Hartford Hospital Evidence-based Practice Center, Hartford, CT 5Department of Obstetrics and Gynecology, Lozano-Blesa University Hospital, Domingo Miral s/n, Zaragoza, Spain.

\begin{abstract}
OBJECTIVE:: We performed a systematic review and meta-analysis of randomized controlled trials (RCTs) assessing the effect of programmed exercise for at least 12 weeks, in postmenopausal women on insulin sensitivity-related outcomes (ISROs), including fasting insulin, C-peptide, insulin growth factor (IGF-1) and IGF-binding protein (IGFBP-3), Homeostatic Model Assessment-Insulin Resistance (HOMA-IR), and anthropometric variables. METHODS:: Searches were conducted in PubMedMedline, Embase, Scopus, Web of Science, and Cochrane Library from inception through May 3, 2016, for studies published in all languages. Extracted data included characteristics of the study design, study participants, intervention, and outcome measures. Types of exercise were classified into "mid-term exercise intervention" (MTEI, 3-4 months exercise duration) and a "long-term exercise intervention" (LTEI, 6-12 months exercise duration). Risk of bias in RCTs was evaluated with the Cochrane tool. We used random-effects models for metaanalyses. We adhered to the Preferred Reporting Items for Systematic Reviews and Meta-Analyses guidelines. RESULTS:: Seven RCTS ( $n ?=$ ?580) evaluating the effects of programmed exercise on ISROs were included. In three RCTs, MTEI
\end{abstract}


significantly lowered insulin levels (mean difference [MD] -6.50?pmol/L, 95\% confidence interval $[\mathrm{Cl}]-11.19,-1.82, \mathrm{P} ?=? 0.006)$ and $\mathrm{HOMA}-\mathrm{IR}$ values (MD $-0.18,95 \% \mathrm{Cl}-0.34,-0.03, \mathrm{P} ?=? 0.02)$ when compared with controls. LTEI had no significant effect on insulin levels $(P ?=? 0.19)$ or HOMA-IR values $(P ?=? 0.68)$ in four and three RCTs, respectively. There were no significant differences between exercise intervention versus controls in circulating IGF-1, glucose, triglycerides with both MTEI and LTEI, and in IGFBP-3 with LTEI. There were significant reductions in body mass index $(\mathrm{BMI}, \mathrm{kg} / \mathrm{m})(\mathrm{MD}-1.48,95 \% \mathrm{Cl}-2.48,-0.48, \mathrm{P} ?=? 0.004)$ and in body fat percentage (MD $-2.99,95 \% \mathrm{Cl}-4.85,-1.14, \mathrm{P} ?=? 0.01)$ after MTEl; and in waist circumference after both MTEI (MD $-1.87,95 \% \mathrm{Cl}-3.02,-0.72$, $P ?=? 0.001)$ and LTEI (MD $-3.74,95 \% \mathrm{Cl}-6.68,-0.79)$. Heterogeneity of effects among studies was moderate to low. CONCLUSION:: Exercising for 3 to 4 months significantly lowered insulin levels and HOMA-IR values, BMI waist circumference, and percentage body fat mass; exercising for 6 to 12 months lowered waist circumference in postmenopausal women. (c) 2017 by The North American Menopause Society.

- ISSN: 10723714

- CODEN: MENOF

- Source Type: Journal

- Original language: English

- DOI: $10.1097 / G M E .0000000000000936$

- Document Type: Article in Press

- Publisher: Lippincott Williams and Wilkins

\section{References}

Bueno-Notivol, J.; 1Facultad de Medicina, Universidad de Zaragoza, Zaragoza, Spain 2ProEd Communications Inc., Cleveland, OH 3School of Medicine, Universidad Peruana de Ciencias Aplicadas (UPC), Lima, Peru 4University of Connecticut/Hartford Hospital Evidence-based Practice Center, Hartford, CT 5Department of Obstetrics and Gynecology, Lozano-Blesa University Hospital, Domingo Miral s/n, Zaragoza, Spain., (C) Copyright 2017 Elsevier B.V., All rights reserved. 